\title{
Kluver-Bucy Syndrome: \\ A Rare Complication of Herpes Simplex Encephalitis
}

\author{
Raquel Costa, Joana Fontes, Tiago Mendes, Marta Pereira, Carlos Gonçalves \\ Unidade Local Saúde Alto Minho, Hospital Conde de Bertiandos, Ponte de Lima, Portugal \\ Doi: 10.12890/2021_002725 - European Journal of Case Reports in Internal Medicine - @ EFIM 2021
}

Received: $17 / 06 / 2021$

Accepted: $21 / 06 / 2021$

Published: $22 / 07 / 2021$

How to cite this article: Costa R, Fontes J, Mendes T, Pereira M,Gonçalves C. Kluver-Bucy syndrome: a rare complication fo Herpes symplex encephalitys. EJCRIM 2021;8: doi:10.12890/2021_002725.

Conflicts of Interests: The authors declare there are no competing interests.

This article is licensed under a Commons Attribution Non-Commercial 4.0 License

\section{ABSTRACT}

Kluver-Bucy syndrome (KBS) is a characterized by a group of cognitive dysfunctions that include hypersexuality, placidity, hyperorality, memory deficits and hypermetamorphosis. This syndrome is often seen in pathological states that destroy the temporal lobes, normally bilaterally. Herpes simplex encephalitis (HSE) is one of the causes of KBS, as the herpes virus can cause dysfunction/destruction of the temporal lobes. KBS is a very rare syndrome, with just a few cases described in the literature. We present the case of a 21-year-old-man who was diagnosis with KBS after HSE.

\section{LEARNING POINTS}

- Kluver-Bucy syndrome (KBS) should be kept in mind in patients with visual agnosia, hypermetamorphosis, memory disturbances, hypersexuality and placidity.

- Neurological causes of KBS should be treated quickly.

- Treatment of KBS is still very challenging.

\section{KEYWORDS}

Kluver-Bucy, herpes virus encephalitis, hypersexuality, hyperorality

\section{INTRODUCTION}

Kluver-Bucy syndrome (KBS) is a characterized by a group of cognitive dysfunctions that include hypersexuality, emotional behavioural changes (especially placidity), hyperorality, memory deficits and hypermetamorphosis (increased interest in every object that enters the visual field) ${ }^{[1,2] .}$ KBS was first described in humans (the first diagnosis was made in monkeys in 1937) in a patient who had undergone bilateral temporal lobectomy for epilepsy in $1955^{[3]}$. The aetiology of KBS is varied and includes herpes simplex encephalitis (HSE), temporal lobe epilepsy, Alzheimer's disease, head trauma, toxoplasmosis, Pick's disease and cerebrovascular disease ${ }^{[4,5]}$. The common pathology of these aetiologies is bilateral destruction or dysfunction of the medial temporal lobe ${ }^{[2,5]}$.

We present the case of a 21-year-old man who developed KBS following HSE, which is very rare.

\section{CASE DESCRIPTION}

We report the case of a 21-year-old man with no relevant medical history. He was on his cell phone when he experienced a generalized tonic-clonic seizure. He was brought to the emergency department (ED) and his mother reported that he had been experiencing high fever, 
headache, nausea and vomiting, with 4 days of evolution. In the ED, he was not aware of what had happened and also had another seizure. The seizures ceased when he was given $10 \mathrm{mg}$ of diazepam; he was also medicated with $1 \mathrm{~g}$ of levetiracetam. He was very agitated, without focal neurological deficits, meningeal signs or fever. Head tomography was normal but showed low cerebellar tonsils. Analysis revealed leucocytosis of $16.3 \times 10^{9}$ with a negative C-reactive protein. Alcohol levels were also negative and urine analysis did not show any drugs of abuse or infection.

Twelve hours later, the patient developed fever, presented with prostration, could follow only simple orders and his speech was meaningless. Because the CT scan showed low cerebellar tonsils, it was decided not to performed a lumbar punction.

Cranial magnetic resonance imaging (MRI) showed bilateral FLAIR hyperintensities in the temporal-frontal cortices (Fig. 1).

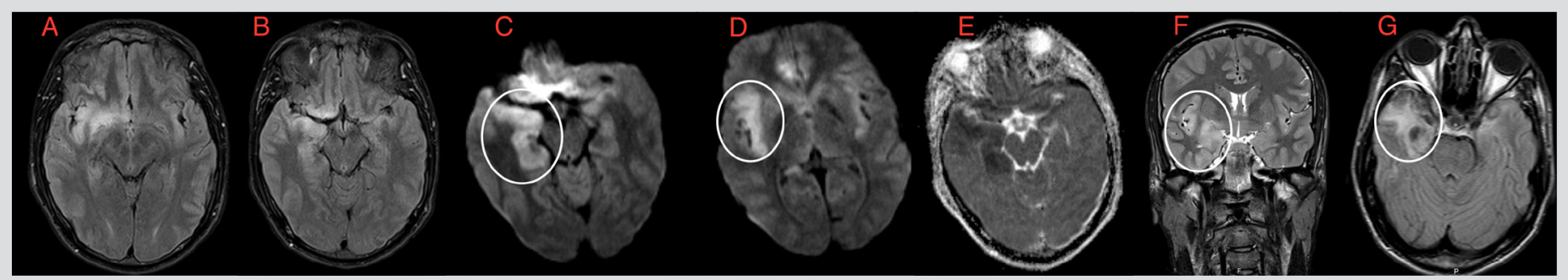

Figure 1. Cranial MRI showing bilateral FLAIR hyperintensities in the temporal-frontal cortices

The most likely diagnosis at this point was meningoencephalitis. He was started on acyclovir (750 mg 8/8 hr), ceftriaxone ( $2 \mathrm{~g} 12 / 12 \mathrm{hr}$ ) and dexamethasone (10 mg 8/8 hr). After the fifth day of hospitalization, the patient was afebrile, without any more seizures. Herpes simplex $1 \mathrm{lgG}$ and IgM antibodies were both positive. The patient's neurological status had improved in terms of awareness, but he began to show behavioural abnormalities, such as dulled emotions (he did not react when another patient was physically agitated), hypersexuality (attempts to touch female staff members), urinating over staff, excessive eating, and eating 'non-food' items (paper towels, faeces). He was diagnosed with Kluver-Bucy syndrome. Risperidone $0.5 \mathrm{mg}$ three times a day, topiramate $50 \mathrm{mg}$ twice a day and fluoxetine $20 \mathrm{mg} / \mathrm{day}$ were initiated. At the time of discharge, he scored 24 on the Mini Mental State Examination (MMSE); although there was some improvement in behaviour, he maintained serious alterations, namely hyperphagia.

At 3-month follow-up, the patient maintained behavioural alterations, such as hypersexuality, hyperphagia and mood swings. The topiramate was suspended and carbamazepine 200 mg every 8 hours was added. Six months later, the hypersexuality and hyperphagia had improved to a mild to moderate level.

\section{DISCUSSION}

Herpes simplex encephalitis can cause both general and focal signs of cerebral dysfunction. The diagnosis is based on clinical findings such as fever, neurological signs (seizures), T2 hyperintensity on MRI and abnormal CSF ${ }^{[6]}$. Herpes simplex virus type 1 accounts for $90 \%$ of HSE. This virus usually has a predilection for the temporal lobes. Acyclovir is the standard treatment for HSE and given for 14-21 days, and is best administered before the patient becomes comatose ${ }^{[4-6]}$. In our patient, CSF analysis was not possible, but the typical changes seen on MRI, fever and seizures made the diagnosis of HSE, which was later confirmed by a positive HSV test.

A patient with KBS following HSE was first described in 1975, but only a few cases of KBS have been reported so far ${ }^{[2]}$. This syndrome can be complete or incomplete and is characterized by visual agnosia (inability to recognize known objects), hypermetamorphosis, memory disturbances, hypersexuality and placidity (decreased motor and verbal reactions in situations that normally cause fear and anger). The incomplete syndrome is more common ${ }^{[1,2]}$. In our case, the patient had all the features except visual agnosia, and so was considered to have an incomplete syndrome.

Treatment for KSB is challenging and as yet, there are no specific medications for this disease. Carbamazepine is useful in behavioural changes like hypersexuality ${ }^{[7]}$. Haloperidol and anti-depressants can also be used. This patient was medicated with fluoxetine, haloperidol and carbamazepine, with some improvement. However, severe behavioural changes remained. The late initiation of acyclovir could have been the cause of this poor prognosis, as it was after the temporal lobes had experienced some destruction.

KBS should be kept in mind in patients with visual agnosia, hypermetamorphosis, memory disturbances, hypersexuality and placidity, especially in those in whom the main aetiology affects the temporal lobes. Quick initiation of treatment can be the difference between a good or a bad outcome. Treatment of KBS is still very challenging. 


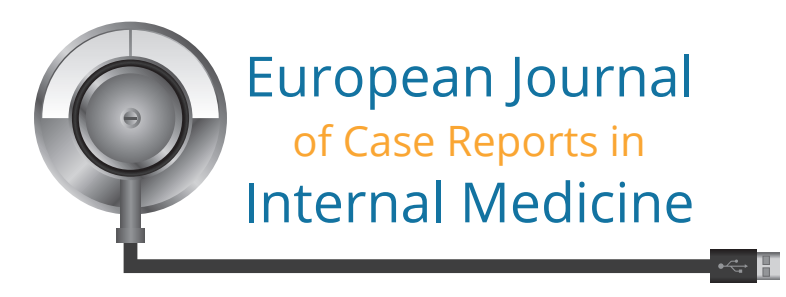

\section{REFERENCES}

Pradhan S, Singh MN, Pandey N. Kluver Bucy syndrome in young children. Clin Neurol Neurosurg 1998;100:254-258.

Mulhan K, Senadim S, Soylemez E, Tekin B, Atakli HD. Kluver-Bucy syndrome following herpes simplex encephalitis. Turk J Neurol 2018;24:165-167.

Terzian H, Ore GD. Syndrome of Kluver Bucy: reproduced in man by bilateral removal of the temporal lobes. Neurology 1955;5:373-380.

Auvichayapat N, Auvichayapat P, Watanatorn J, Thamaroj J, Jitpimolmard S. Kluver-Bucy syndrome after mycoplasmal bronchitis. Epilepsy Behav $2006 ; 8: 320-322$.

Halder A, Mandal U, Halder S, Biswas A. Kluver-Bucy syndrome: a morbid consequence of post herpes simplex encephalitis. CHRISMED J Health Res 2015;2:373-375.

Demaerel PL, Wilms G, Robbercht W, Johannik K, Van Hecke P, Carton H, et al. MRI of herpes simplex encephalitis. Neuroradiology 1992;34:490-493.

7. Stewart JT. Carbamazepine treatment of a patient with Kluver-Bucy syndrome. J Clin Psychiatry 1985;46:496-497. 